\title{
A TEORIA DAADAPTAÇÃO SUSTENTANDO O CUIDADO DE ENFERMAGEM EM HOSPITAL PEDIÁTRICO: UM ESTUDO DE CASO
}

\author{
Regina Aparecida Tremarin ${ }^{1}$, Fabiane Gawleta², Daniele Laís Brandalize Rocha ${ }^{3}$
}

\begin{abstract}
RESUMO: Estudo de caso realizado em um hospital pediátrico de grande porte em Curitiba-PR. O objetivo foi desenvolver cuidado de enfermagem sustentado pelo referencial da Teoria da Adaptação a uma criança hospitalizada e sua família. O estudo foi realizado durante as atividades de ensino clínico, da disciplina de Processo de Cuidar em Enfermagem I, de julho a novembro de 2007. Os modelos teóricos, ao serem utilizados pelos graduandos em Enfermagem como referenciais para a sistematização do cuidado, contribuem para o aprimoramento da prática e a organização, interpretação e análise do processo de cuidar, ampliando o nível de conhecimento na formação de profissionais e na pesquisa de enfermagem. Concluímos que o estudo de teorias de enfermagem e sua aplicação na prática por alunos de graduação devem ser incentivados pelas instituições formadoras para contribuir com a construção do conhecimento em enfermagem e aprimorar a assistência prestada.
\end{abstract}

PALAVRAS-CHAVE: Teoria de enfermagem; Cuidados de enfermagem; Pediatria.

\section{THE ADAPTATION THEORY AS A FOUNDATION TO NURSING CARE AT A PEDIATRIC HOSPITAL: ACASE STUDY}

\begin{abstract}
Grounded on the theoretical framework of the Adaptation Theory, we report a case study held at a large pediatric hospital in the city of Curitiba/Paraná State, Brazil, during the third-term nursing undergraduation clinical studies. The case study was carried out with a hospitalized child and its mother during the clinical teaching activities in the discipline Caring Process in Nursing I. The theoretical models, when used by nursing undergraduates as a framework for care systematization, contribute to practice refinement as well as organization, interpretation and analysis of the caring process, broadening knowledge level in professional education and nursing research. We concluded that the study of nursing theories and its practical use by undergraduates must be encouraged by educational institutions.
\end{abstract}

KEYWORDS: Nursing theory; Nursing care; Pediatrics.

\section{LATEORÍA DE LAADAPTACIÓN SUSTENTANDO EL CUIDADO DE ENFERMERÍA EN HOSPITAL PEDIÁTRICO: UN ESTUDIO DEL CASO}

RESUMEN: Estudio de un caso realizado en un hospital pediátrico de gran porte en la ciudad de Curitiba-PR. El objetivo fue desarrollar cuidado de enfermería sustentado por el referencial de la Teoría de la Adaptación a un niño hospitalizado y su familia. El estudio fue realizado durante las actividades de enseñanza clínica, de la disciplina de Proceso de Cuidar en Enfermería I, de julio a noviembre de 2007. Los modelos teóricos, al ser utilizados por los estudiantes de Enfermería como referenciales para la sistematización del cuidado, contribuyen para el perfeccionamiento de la práctica y la organización, interpretación y análisis del proceso de cuidar, ampliando el nivel de conocimiento en la formación de profesionales y en la investigación de enfermería. Concluimos que el estudio de teorías de enfermería y su aplicación en la práctica por alumnos de graduación deben ser incentivados por las instituciones formadoras para contribuir con la construcción del conocimiento en enfermería y primorear la asistencia prestada.

PALABRAS CLAVE: Teoría de enfermería; Cuidados de enfermería; Pediatría.

\footnotetext{
${ }^{1}$ Acadêmica do curso de Enfermagem da Faculdades Pequeno Príncipe-FPP.

${ }^{2}$ Acadêmica de do curso de Enfermagem da FPP.

${ }^{3}$ Enfermeira. Mestre em Enfermagem pelo Programa de Pós-Graduação em Enfermagem da Universidade Federal do Paraná. Docente do Curso de Enfermagem da FPP.
}

Autor Correspondente:

Daniele Laís Brandalize Rocha

Rua Wanda dos Santos Mullmann, 1266 - 83323-400 - Pinhais-PR

E-mail: enferdani2004@yahoo.com.br 


\section{INTRODUÇÃO}

A doença e hospitalização da criança são marcantes não somente para ela, mas para a família que, juntamente com o filho, são separados do seu mundo familiar e de sua rotina, e passam a conviver em um ambiente novo e desconhecido que é o hospital. Isto acarreta desgaste físico e psicológico para os familiares, os quais podem reagir de diferentes formas, tendo diferentes reações como o medo, a insegurança, entre outros ${ }^{(1)}$. Durante a hospitalização, criança e família se encontram fragilizados, propensos a sofrerem com todas as alterações não somente do novo ambiente, mas também com as intervenções que são realizadas visando o tratamento e cura da criança. Dessa forma, o cuidar em pediatria deve estar diretamente relacionado à criança e ao seu familiar, entendo-se este como extensão paciente e também alvo dos cuidados de enfermagem ${ }^{(2)}$.

Entendemos que o cuidado de enfermagem deve ser sistematizado através de um método, o qual deve ser embasado em um referencial teórico que direcione o olhar e fundamente cientificamente as ações do enfermeiro. Os referenciais teóricos ou modelos teóricos na enfermagem são chamados de Teorias de Enfermagem e representam, atualmente, os esforços coletivos e individuais dos enfermeiros para definir e dirigir a profissão e proporcionar a base para um desenvolvimento teórico continuado ${ }^{(3)}$.

Ao escolher uma teoria de enfermagem para fundamentar e direcionar o cuidado deve ser considerada a adequação e a aplicabilidade à situação de enfermagem em que será utilizado ${ }^{(3)}$. Enquanto acadêmicas de enfermagem tivemos a oportunidade de conhecer, em sala de aula, algumas das principais teorias de enfermagem e, desde o primeiro contato, a Teoria da Adaptação de Roy nos chamou a atenção e nossa inquietação passou a ser como poderíamos aplicá-la em nossa prática de cuidado.

Durante nossa vivência no ensino clínico em pediatria, tivemos a oportunidade de cuidar da criança doente e hospitalizada e sua família, na qual percebemos que, além da criança, esta necessitava de cuidados visando o atendimento de suas necessidades e cura da doença. $\mathrm{O}$ familiar, mais especificamente a mãe, ao permanecer ao lado do filho durante a hospitalização, passava por essa vivência de forma intensa, apresentando modificações em sua vida pessoal e familiar, necessitando, assim, de cuidados de enfermagem.
Escolhemos a Teoria da Adaptação de Roy para direcionar o nosso cuidado à criança e família, enquanto acadêmicos de enfermagem, considerando nossa afinidade com seus principais conceitos e sua possível aplicabilidade nesse contexto vivido. Sendo assim, conduzidas pelo referencial teórico adotado e pelo objetivo colocado para o desenvolvimento deste estudo, buscamos compreender os aspectos teórico-práticos da enfermagem, os quais são indispensáveis para o desenvolvimento do cuidado.

Consideramos que o conhecimento teórico influencia a realidade e este adicionalmente no desenvolvimento das teorias tornando-se importante para guiar a educação, a pesquisa e a prática. Apesar de toda a evolução da ciência e, consequentemente, da enfermagem, ainda se percebe certa dificuldade por parte dos enfermeiros em trabalhar com teorias, tanto no âmbito da assistência, quanto no do ensino ou pesquisa. Com vistas à superação desta dificuldade, destaca-se a importância da iniciativa da aplicação de marcos teóricos durante o período acadêmico para uma melhor construção do conhecimento em enfermagem, no intuito de aprimorar a assistência prestada.

Neste estudo tivemos como objetivo desenvolver o cuidado de enfermagem sustentado pelo referencial da Teoria da Adaptação a uma criança hospitalizada e sua família.

\section{A TEORIA DA ADAPTAÇÃO DE ROY}

De acordo com Roy, a pessoa, como um sistema, tem a capacidade de se adaptar e criar mudanças no meio ambiente. Sendo um sistema, a pessoa recebe estímulos, dentre eles o focal, que é o estímulo interno ou externo que constitui o maior grau de mudança, gerando um forte impacto. Os estímulos contextuais, que são todos os outros estímulos presentes na situação que contribuem para o efeito do estímulo focal e os estímulos residuais, que são os fatores cujos efeitos na situação atual não são centrais e a pessoa pode não ter consciência da influência destes fatores. São fatores descentralizados da situação atual, mas que a influenciam. A vivência dos estímulos é simultânea, ocorrem sem uma ordem aparente, podem ocorrer ao mesmo tempo, sem que a pessoa se dê conta que estão atuando ${ }^{(4)}$.

No contexto de doença e hospitalização, criança e familiar recebem estímulos, em que o principal estímulo, o focal, que gera o maior impacto, é a própria 
doença; os estímulos contextuais são todos os outros fatores, como mudança de ambiente e rotinas de vida, separação dos demais familiares. O conhecimento que se tem da doença, o apoio que criança e familiar recebem, entre outros, que vão acentuar ou atenuar os efeitos do estímulo focal, na criança e no familiar que a acompanha. Os estímulos residuais serão os demais estímulos, os quais não se relacionam diretamente com a vivência atual da doença e hospitalização, mas têm influência sobre ela, como experiências anteriores com hospitalização, doença na família.

De acordo com a Teoria da Adaptação de Roy, cada pessoa, como um sistema adaptativo, tem um nível de adaptação, o qual é determinado por processos de controle, também chamados de mecanismos de enfrentamento reguladores (fisiológico) e cognatos (sentimentos e mobilizações emocionais) os quais são subsistemas da pessoa como um sistema adaptativo. Sendo assim, ao receber estímulos (focal, contextuais e residuais), iniciam-se, na pessoa, processos de controle, em que os mecanismos de enfrentamento irão determinar os comportamentos a serem expressos, podendo ser adaptáveis ou não(4).

Acreditamos que, criança e familiar, como um sistema, têm a capacidade de se adaptar à nova situação, a qual é permeada pela ocorrência de inúmeros estímulos. O comportamento apresentado por ambos, frente aos mesmos estímulos, dependerá dos mecanismos de enfrentamento ou processos de controle individuais e nem sempre, consegue-se chegar à adaptação, tendo-se inúmeros comportamentos ineficazes.

A investigação do comportamento da pessoa pode ser realizada nos quatro modos adaptativos identificados por Roy, os quais resultam dos mecanismos de controle reguladores e cognatos, são eles: fisiológico, autoconceito, função de papel e interdependência. A mobilização dos quatro modos de adaptação é que configuram a intensidade dessa vivência da criança e familiar, pois se alteram de acordo com a experiência singular de cada um com maior ou menor intensidade ${ }^{(4)}$.

O modo de adaptação fisiológico é facilmente percebido, pois envolve as necessidades básicas do subsistema regulador, porém os demais modos são subjetivos e vivenciados de maneira diferente, mesmo diante do mesmo estímulo. O modo de autoconceito está relacionado aos aspectos psicológicos e espirituais da pessoa, envolve o eu físico e o eu pessoal; o modo de função de papel envolve os padrões de interação social e o modo de interdependência, os relacionamentos interpessoais, as necessidades afetivas, como valor humano, afeição, amor, afirmação( $^{(4)}$.

O objetivo dos cuidados de enfermagem à criança e familiar nessa vivência deve ser promover a adaptação e ajudá-los a lidar com os problemas de adaptação gerados. Suas ações devem visar controlar os estímulos focais, contextuais ou residuais que estão sobre eles. O enfermeiro deve agir preparando-os para as mudanças antecipadas através do fortalecimento dos mecanismos de enfrentamento regulador e cognitivo individuais ${ }^{(5)}$.

Compreender a experiência da criança doente e hospitalizada e familiar que a acompanha aponta para um cuidado de enfermagem que contemple a criança e familiar, tendo em vista o ser humano como um ser integral, com sentimentos, conflitos e vivência e considerando o impacto que essa vivência gera em $\operatorname{ambos}^{(6)}$. Acreditamos que é responsabilidade do enfermeiro ajudá-los nessa adaptação, pela identificação do nível de adaptação e da necessidade de intervenção de enfermagem nos quatro modos de adaptação. Esta intervenção deve ser realizada pelo enfermeiro por meio da aplicação do Processo de Enfermagem, o qual, conforme o modelo de adaptação de Roy consiste em seis passos: avaliação dos comportamentos, avaliação dos estímulos, diagnósticos de enfermagem, estabelecimento de objetivos, intervenções e avaliação ${ }^{(4)}$.

Acreditamos que a Teoria da Adaptação de Roy aplica-se a essa realidade e direcionar o processo de enfermagem embasando-se cientificamente nessa teoria possibilita ao enfermeiro a auxiliar criança e familiar a apresentar comportamentos eficazes, que contribuam para a sua adaptação.

\section{METODOLOGIA}

Após o estudo da teoria e embasados em seus conceitos, procuramos aplicá-la em nossa prática, durante os momentos de ensino clínico da disciplina de Processo de Cuidar em Enfermagem I, do $3^{\circ}$ período do Curso de Graduação em Enfermagem, ocorrido nos meses de julho a novembro de 2007. Realizou-se o processo de enfermagem de acordo com o modelo da Teoria de Adaptação de Roy, em uma criança doente e hospitalizada e seu familiar, mais especificamente a mãe, que a acompanhava em uma 
unidade de internação clínica de um hospital pediátrico de grande porte de Curitiba. A escolha por estes sujeitos deveu-se à aleatoriedade, pois, ao realizarmos as ações de cuidado durante o ensino clínico nos aproximamos desta criança e sua mãe e realizamos o processo de enfermagem, orientados pela professora supervisora de estágio.

No desenvolvimento deste trabalho foram respeitados o Código de Ética profissional e a Resolução n. ${ }^{\circ}$ 196/96 que levam em conta princípios éticos básicos que devem orientar os estudos envolvendo seres humanos $^{(7)}$. O estudo iniciou-se após o consentimento da instituição, oficializado pela coordenação da disciplina de Processo de Cuidar em Enfermagem I, do Curso de Graduação em Enfermagem de uma Faculdade particular na cidade de Curitiba.

$\mathrm{O}$ cuidado à criança e à mãe foi sustentado na Teoria da Adaptação de Roy e percorreu todas as fases do processo de enfermagem conforme proposto pela teoria, o qual passamos a descrever:

No primeiro passo do processo de enfermagem o enfermeiro deve recolher dados sobre os comportamentos da criança e familiar e o estado atual da adaptação. O comportamento é o único indicador de como estão se adaptando às mudanças. O convívio e a proximidade permitem ao enfermeiro avaliar, detectar necessidades diante de distintos estímulos a que estão expostos. Na segunda fase o enfermeiro deve avaliar os estímulos externos e internos que estão influenciando os comportamentos em cada modo adaptável. Depois de identificados os estímulos, eles devem ser classificados em focais, contextuais e residuais. Na terceira fase, os dados comportamentais, uma vez reunidos, devem ser interpretados. Nesta interpretação o enfermeiro deverá considerar os comportamentos juntamente com os outros fatores, isto é, os estímulos que afetam o seu comportamento e estabelecer os diagnósticos de enfermagem.

$\mathrm{Na}$ quarta fase do processo, enfermeiro estabelece os objetivos, que é a determinação de afirmações claras de resultados comportamentais esperados a partir dos cuidados de enfermagem. $\mathrm{O}$ objetivo geral das intervenções de enfermagem é manter ou aumentar o comportamento adaptável e modificar o comportamento ineficaz tornando-o adaptável. Na quinta fase, logo após estabelecer os objetivos relativos aos comportamentos que irão promover a adaptação, o enfermeiro deverá determinar como intervir para auxiliar para que criança e familiar atinjam estes objetivos. A intervenção de enfermagem irá determinar como os objetivos estabelecidos serão obtidos. Por fim, na última fase do processo de enfermagem, o enfermeiro verifica a eficácia da intervenção de enfermagem em relação ao comportamento, isto é, se o objetivo estabelecido foi atingido.

\section{RESULTADOS}

O cuidado à criança e mãe foi realizado conforme as fases do processo de enfermagem proposto por Roy, conforme descrevemos a seguir:

Na primeira fase, que é a avaliação dos comportamentos, investigamos o comportamento da criança e mãe nos quatro modos de adaptação. No modo fisiológico observamos as respostas que ambos apresentavam: relacionadas à nutrição, sono e repouso, eliminação; no modo do autoconceito, buscamos descobrir como estavam se sentindo frente a essa situação e se essas questões geraram alguma forma de distúrbio tanto no seu eu físico, relacionado à sua imagem corporal, quanto no seu eu pessoal, relacionado às suas próprias características, expectativas e valores; no modo de função do papel, procuramos identificar como estavam se relacionando com as pessoas ao seu redor, como se conformava a rede de apoio familiar e social, se de alguma forma a doença e hospitalização gerou algum déficit em seu relacionamento social. E no modo de interdependência, buscamos descobrir se as necessidades afetivas estavam sendo preenchidas, se houve déficit de afeição, amor e afirmação, sentimentos de tristeza ou depressão, ou se estavam passando por períodos de isolamento, solidão e alienação.

Após a avaliação dos comportamentos, passamos para a segunda fase, que é a avaliação dos estímulos. Para a avaliação do estímulo focal, procuramos analisar o impacto, na criança e mãe, desde o primeiro dia de hospitalização, com os sentimentos que foram mobilizados, a trajetória percorrida desde a descoberta da doença até a hospitalização e tratamento da criança, a expectativa futura sobre a sua recuperação.

$\mathrm{Na}$ avaliação do estímulo contextual, procuramos o que mais estava a contribuir para o efeito do estímulo focal e fizemos questões sobre a cultura (nível sócio-econômico, etnia, sistema de crenças), a família (estrutura, tarefas), estado de desenvolvimento da criança (idade, sexo, tarefas, hereditariedade e fatores genéticos), eficácia cognitiva (percepção, conhecimento, qualidades) e considerações ambientais (mudanças no ambiente externo e interno). $\mathrm{Na}$ avaliação dos estímulos residuais buscamos os demais 
fatores que estavam influenciando indiretamente a situação atual, como experiências passadas de situações similares já vivenciadas.

Após a avaliação dos comportamentos e estímulos, passamos para a terceira fase, na qual realizamos os diagnósticos de enfermagem. De acordo com o proposto na Teoria de Adaptação de Roy, uma das formas estabelecidas para a definição de um diagnóstico de enfermagem envolve posicionar o comportamento juntamente com os estímulos mais relevantes e foi dessa forma que procuramos elaborar todos os diagnósticos de enfermagem.

Por exemplo, na avaliação da criança: apresentava face em forma de lua (comportamento), consequente ao uso de corticóides (estímulo). Dessa forma o diagnóstico de enfermagem formulado foi: Face em forma de lua relacionada ao uso de corticoides. Na avaliação da mãe: apresentava agitação, insônia, nervosismo e apreensão (comportamentos). Todos estes comportamentos indicam a ansiedade e resultam de um estímulo focal, que é a vivência da doença e hospitalização do filho. Dessa forma o diagnóstico de enfermagem formulado foi: Ansiedade relacionada à vivência da doença e hospitalização do filho. Assim, foram formulados todos os diagnósticos de enfermagem relacionados aos comportamentos da criança e mãe e aos estímulos que os influenciavam.

Formulados os diagnósticos de enfermagem, passamos para a quarta fase do processo, que é o estabelecimento de objetivos. Nessa fase fizemos a determinação de afirmações claras de resultados comportamentais que esperávamos, a partir dos cuidados de enfermagem para ambos. O objetivo geral para as intervenções de enfermagem é manter ou aumentar o comportamento adaptável e modificar o comportamento ineficaz tornando-o adaptável.

Por exemplo, para os diagnósticos de enfermagem acima citados, o objetivo esperado dos cuidados de enfermagem foi o de minimizar o impacto que a doença, hospitalização e as alterações na condição física da criança provocaram na mãe (como a alteração no formato da face da criança).

Formulados todos os objetivos para os cuidados de enfermagem, passamos para a quinta fase do processo, que é o estabelecimento de intervenções. Nesta fase, procuramos alterar, aumentar, diminuir, remover ou manter os estímulos, visando aumentar a capacidade da criança e mãe em responder positivamente, apresentando respostas adaptáveis.
Seguindo o exemplo acima citado, de imediato não se pode remover o estímulo focal, que é a própria doença da criança, mas pode-se trabalhar com os estímulos contextuais e residuais que estão a influenciá-los. Por exemplo, conversar com a mãe, ouvir suas dificuldades e expectativas, motiva-la a sentir-se fortalecida; esclarecer as dúvidas e inseguranças que a mãe tem em relação ao estado de saúde da criança; permitir que a mãe faça a troca com outro familiar no acompanhamento da criança, para que possa descansar; promover o brinquedo terapêutico, cuidando da criança a partir do lúdico, entre outros.

Por fim, chegamos à última fase do processo que é a avaliação. Finalizando o exemplo já seguido anteriormente para ilustrar nosso cuidado, utilizamos a observação da criança e a conversa com a mãe para avaliar a eficácia das intervenções que havíamos realizado, já que tivemos a oportunidade de voltar à unidade de internação no dia seguinte. Percebemos que os cuidados/intervenções que havíamos realizado com a criança e mãe haviam sido positivos, já que ambos foram receptivos a nós, a mãe apresentava-se menos ansiosa e segura, sendo que o seu relacionamento com a criança ficou fortalecido, já que ela não apresentava mais a insegurança citada anteriormente.

\section{CONSIDERAÇÕES FINAIS}

Considerando que a adoção de um referencial teórico para realizar a assistência de enfermagem se faz necessária para o aprimoramento da qualidade do cuidado. A utilização da Teoria da Adaptação de Roy nos permitiu acessar de forma mais individualizada, organizada e integral as necessidades individuais da criança e sua mãe em seu próprio contexto e nas circunstâncias de vida no momento da hospitalização.

Após o término do estudo, verificamos que a hospitalização, a qual traz consequências à criança e à sua mãe, pode ser caracterizada como um período de grande investimento de energia para enfrentar e adaptar-se ao novo papel que o adoecer estabelece. A criança hospitalizada habita o mundo hospitalar, vivencia sentimentos muitas vezes desconhecidos, entre eles o de dor e de angústia e, neste contexto, a participação familiar, em especial da mãe, é muito importante. Porém, nesta vivência, a mãe também passa por enfrentamentos, mudanças na vida cotidiana, além de surgirem inúmeros questionamentos sobre a doença e recuperação do filho hospitalizado. Dessa forma, os sistemas adaptativos da criança e mãe, muitas 
vezes não são suficientes para que ambos enfrentem e respondam positivamente aos estímulos.

Neste sentido, percebemos o papel fundamental do enfermeiro que, ao se embasar na Teoria de Adaptação de Roy no cuidado das crianças doentes e hospitalizadas e seus familiares, ao desenvolver intervenções, pode auxiliar na adaptação à condição que lhes foi imposta.

O estudo ora desenvolvido nos permitiu um olhar reflexivo e crítico sobre a utilização de teorias de enfermagem e contribuiu para o desvelar de uma realidade até o momento pouco conhecida para nós, o cuidar em pediatria. Durante esta experiência, buscamos transpassar as barreiras e limitações próprias de alunos da graduação em enfermagem, ou seja, a timidez diante do paciente, a falta de destreza na realização de procedimentos e técnicas, como a de exame físico da criança. Além disso, lançar o olhar sobre a realidade de cuidado tendo como direcionador uma teoria de enfermagem não foi fácil e exigiu de nós leituras e releituras da teoria e seus principais conceitos, a fim de nos revestirmos dela, incorporarmos sua proposição, para que, ao nos depararmos com a realidade do cuidado, pudéssemos refletir e estar aptos a iniciar a associação, tão esperada, da teoria com a prática.

Acreditamos que, ao compartilhar este exercício, podemos contribuir para que os demais acadêmicos de enfermagem e os enfermeiros envolvidos no tratamento da criança e sua família reflitam sobre a sua prática de cuidar, engajando-se na busca constante de maneiras e perspectivas para uma assistência de enfermagem holística e científica.

Enquanto acadêmicos de enfermagem, concluímos que, realizar um estudo à luz de uma teoria, especificamente a Teoria da Adaptação de Roy, veio enriquecer nosso conhecimento técnico-científico, tendo aprofundado nossos conceitos e nos levado a refletir sobre o cuidado dispensado a criança hospitalizada e sua família. Os modelos teóricos, ao serem utilizados pelos graduandos em Enfermagem como referenciais para a sistematização do cuidado, contribuem para o aprimoramento da prática e a organização, interpretação e análise do processo de cuidar, ampliando o nível de conhecimento na formação de profissionais e na pesquisa de enfermagem.

Acreditamos que esse estudo pode contribuir para a melhoria da qualidade do cuidado de enfermagem à criança enquanto hospitalizada, a partir da identificação de comportamentos ineficazes desta, propiciando a criação de intervenções que facilitem a sua convivência no hospital e aprimorando orientações a serem fornecidas para a mãe, visando a sua adaptação. Sendo assim, o estudo de teorias de enfermagem e sua aplicação na prática por alunos de graduação devem ser incentivados pelas instituições formadoras.

\section{REFERÊNCIAS}

1. Forte TL, Sato CM. Programa família participante: a humanização hospitalar como resgate da dignidade, exercício da cidadania e transformação da gestão hospitalar. Curitiba: Associação Hospitalar de Proteção a Infância Dr. Raul Carneiro/ Hospital Pequeno Príncipe, 2006.

2. Cordeiro CCK. O cuidado de enfermagem à família de crianças hospitalizadas diante da cronificação da doença [monografia]. Curitiba (PR): Instituto de Ensino Superior Pequeno Príncipe; 2006.

3. Westphalen MEA, Carraro TE, organizadores. Metodologias para assistência de enfermagem: teorizações, modelos e subsídios para a prática. Goiânia: AB; 2001.

4. Roy SC, Andrews HA. Teoria da enfermagem. O modelo de adaptação de Roy. Lisboa: Instituto Piaget; 2001.

5. George JB, Belcher JR, Bennett AM, Crane MD, Cross JR, Falco SM, et al. Teorias de enfermagem: os fundamentos à prática profissional. $4^{\mathrm{a}}$ ed. Porto Alegre: Artmed; 2000.

6. Brandalize DL. O Cuidado de enfermagem à mãe na vivência do processo de transição de saúde-doença do filho com cardiopatia congênita [dissertação]. Curitiba (PR): Universidade Federal do Paraná; 2007.

7. Ministério da Saúde (BR). Conselho Nacional de Saúde. Diretrizes e normas regulamentadoras de pesquisa em seres humanos. Resolução n ${ }^{\circ}$ 196, de 10 de outubro de 1996. Brasilia; 1996. 